\title{
CYCLIN D1: As a Risk of Breast Cancer
}

\author{
*Preeti Chauhan ${ }^{1}$, Palki sahib kaur ${ }^{2}$, Arockia M Babu ${ }^{3}$ \\ 1,2 Department of Biotechnology, Chandigarh College of Technology, Landran, 140307, Mohali, Punjab, India \\ 3 Chandigarh College of Pharmacy, Landran, 140307, Mohali, Punjab, India \\ Email Id: *preetidevi.4406@cgc.edu.in
}

\begin{abstract}
Breast cancer is varying group of tumors in terms of disease prognosis. Breast cancer is found as second main cause of death related with cancer. Cyclin D1 (CCND1) is an important regulator of cell cycle. CCND1over expressed in various human cancers like head, oesophageal, neck, squamous, colon, breast, prostateetc. Many studies have conducted to find correlation between polymorphism in CCND1 gene and development of breast cancer. Some studies found certain genotypes related with risk of breast cancer development. Some studies also proposed the role of varying genetic backgrounds in breast cancer development. This review focuses on role of CCND1 polymorphism and possible risk factors associated with breast cancer, which may explicate the breast cancer screening, prevention, and treatments strategies for future use.
\end{abstract}

Indexed Terms- : Cyclin D1, breast cancer, polymorphism, cell cycle. (Keywords)

\section{INTRODUCTION}

Breast cancer is varying group of tumours in disease prognosis. Breast cancer is found as second main cause of death related with cancer[1].Cell cycle proceeds by the activation of CDK (cyclin dependent kinase)which are controlled by various cyclins and inhibitors of cyclin dependent kinases [2,3].CDK (Cyclin Dependent Kinase) is a heterodimer and made up of catalytic subunit i.e. regulatory subunit known as cyclin and cyclin dependent kinases.

Activity of CDK is generally decided by binding of regulatory cyclin subunit, as positive and negative regulatory phosphorylation $[3,4]$. Cyclin/CDK complexes phosphorylatethe substrates those are required for progression of cell cycle process. Stimulation of growth factor speed up the cell cycle progression which is requiredonly up to a point in G1 phase of cell cycle. This phase isknown as point of restriction [4].

Ahead of this point, cells are mainly committed to only a simple round of cell proliferation without referring the presence of any mitogen or carcinogens. Progression through the G1 phase needs the activities of D and E type cyclinin relation with their kinase subunits [5]. The D-type Cyclini.e. D1, 2, 3, CDK4 or CDK6 acts as target during cancer formation. Over expression of Cyclin D1 is found as most frequently associated with breast cancer development out of all cyclin families.

Frequency of cyclinD1 variation has been found to be connected with risk of other cancers also including, oral, cervical, oesophageal, urinary bladder, lung, colorectal and prostate[6,7]. The conclusions of these studies were inconsistent in different ethnic groups. To study these encounters in detail, many researches have been executed worldwide to check effect of CCND1 polymorphism and risk for different types of cancer [8,9]. This review shows role of CCND1 as an oncogene for cell division machinery especially in breast cancer cases.

\section{II.CELL CYCLE ASSOCIATION WITH BREAST CANCER DEVELOPMENT}

two main stages: mitosis i.e. nuclear division phase and inter phase.

Cell cycle progression occurred by activation of cyclindependent kinases (CDKs).Activated CDKand cyclin complexes lead to phosphorylation and then inactivation of retinoblastoma (' $\mathrm{RB}$ ')protein [10] (fig. 1). The process is regulated by many inhibitors (CKIs), which are divided into two groups known as: cyclin kinase 4 inhibitors (INK4) and CDK inhibitor protein (KIP /CIP).

INK4 groups includes CDKN2A (p16 ARF/p14//INK4A/),CDKN2B (INK4B /p15), CDKN2C (p18/INK4C/)and CDKN2D (p19/INK4D). These groups are associate with CDK4 \& CDK6 and then further interfere its interaction with other subunit of cyclin $\mathrm{D}$. The KIP /CIP group consists ofCDKN1A (p21/WAF1 /CIP1) and CDKN1B (p27/KIP1) which form a trimetric complexes with the G1 transition cyclin dependent kinase. It does not affect the further binding of cyclin [11] (fig. 2).

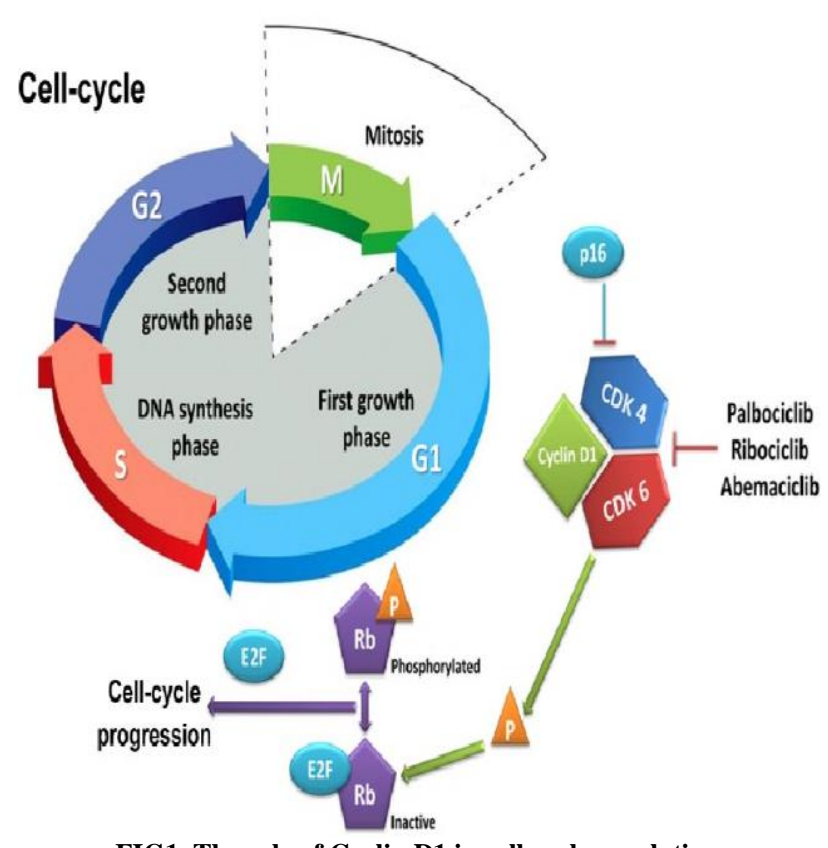

FIG1. The role of Cyclin D1 in cell cycle regulation

Division of cell is comprises of two successive processes i.e. chromosomes segregation into two different cells and DNA replication. Cell division is divided into 


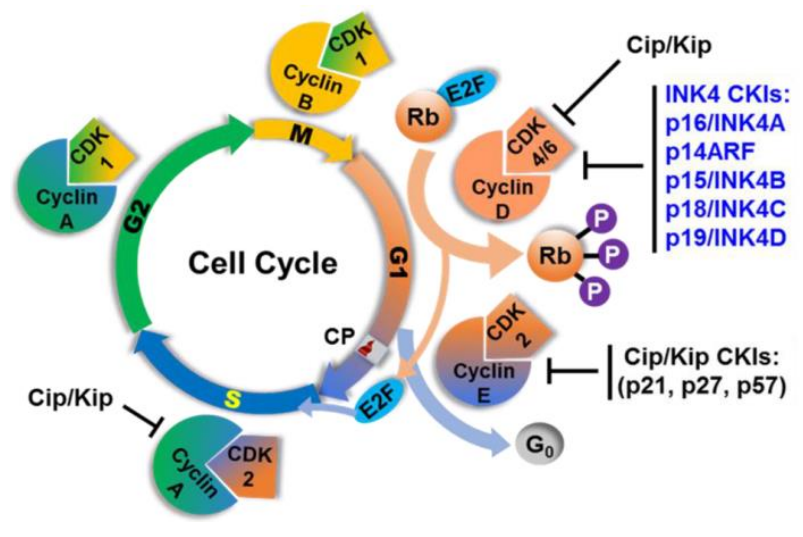

FIG 2.Association of cyclin kinase 4 inhibitors (INK4) and CDK inhibitor protein (KIP /CIP) in cell cycle regulation as well as breast cancer development

Loss of expression of CDKN1B is also important in breast cancer development and is associated with poor prognosis of disease and high tumour grade [12].Some studies noted the importance of CDKN1B C79T polymorphism withrisk of breast cancer risk $[11,13]$.

CCND1 is a member of Dtype cyclins which control G1 cell cycle phase. Increased expression of CCND1 prolongs this phase, and disturbs normal control oncell cycle. This enhances the development as well as progression of breast cancer[14]. In some studies it is found that CCND1 is related with other cancers like colorectal, gastric, breast, prostateetc.[15].

\section{III.CYCLIN D1(CCND1)}

Cyclin D1 protein is encoded by the CCND1 gene. It is present on long arm of chromosome no. 11q13. Cyclin D1 protein is important forproliferation, differentiation, and transcriptionof cell [16].Many studies observed a linkage between the CCND1 870A allele and elevated risk of development of breast cancer $[11,16]$.

Cyclin D1 acts by phosphorylation that leads to inactivationof RB protein in connection with CDK4 (cyclin dependent kinase 4), which promote the transcription factor (TF) release known as E2F from RB (retinoblastoma protein). This will start the process of DNA synthesis[17].Over expression of cyclin D1 result in phosphorylation of retinoblastoma (RB) and then uncontrolled growth of cells[17].

Cyclin D1 is present mainly in two forms i.e. cyclin D1a \& cyclin D1b. Cyclin D1b form is devoid of residues which are required for its export through nucleus. Cyclin D1b ispresentin nucleus, with an increased transforming capacity as comparison tocyclin D1a, which is a nuclear oncoprotein[18].Polymorphisms in CCND1 don't lead to the change in amino acid but it enhances the expression of cyclin D1b, an alternative transcript encoding [16].

CCND1 gene isone of the most over expressed oncogenes in breast carcinoma. Around $45-50 \%$ of IDC (invasive ductal carcinomas) occurs due to over expression of only this oncoprotein [14]. Alteration in CCND1 gene is the very first step in breast cancer formation. Polymorphism in CCND1 has also been found in correlation with breast cancer progression [19].

\section{CYCLIN D1 ASSOCIATION WITH CLINICOPATHOLOGICAL PARAMETERS}

In clinicopathological parameters like tumor size, grade, lymph nodes (LN) status and expression of estrogen, progesterone receptors (ER and PR) Her2/neu etc. was studied by many researchers. Tumor grade, size as well as lymph node (LN) status was notfound correlated with expression of Cyclin D1 in some studies [20,21]. Positive expression of estrogen and progesterone found significantly correlated with cyclinD1 expression in previous studies especiallyin invasive breast carcinoma[21,22]. These results pointed out the role of estrogen and progesterone receptor mediated role of cyclin D1 [23].

In some studies it was notes that low tumor grade, estrogen, progesterone receptor (positive) and Ki67 expression showed statistically significant correlation with cyclin D1[23]. However lymph node status, tumor size and Her2/neu expression were not associated with cyclin D1 expression[23].Some studies found positive correlation of cyclin D1 with lymph node status and negative correlation with Her2/neu and p53 expression especially in luminal B type tumors. However, the expression of cyclin D1 was missing in basal type of tumor cells [24].

Over expression of cyclin D1 has been linked with breast cancer that over express human epidermal growth factor receptor 2 which are generally estrogen receptor (ER) negative. Firstly, it was recommended that CD Kinase function of CCND1 solely leads to its progression into G1 phase. This process caused cell differentiation and lead to its potential of on cogenicity. However, proof from several clinical studies fails to upkeep this hypothesis, and therefore, a different CDK-independent function resulting in carcinogenesis had been suggested[25].

CCND1 is over expressed mainly in those breast cancer cells which show estrogen positive receptors. It has been recommended that alteration in transcriptionvia its action on estrogen receptormay be responsible for carcinogenic potential of CCND1 in breast cancer. This proposition is governed by various outcomes which show connection between CCND1 and estrogen receptor that activates estrogen regulated genes in estrogennegative cases [26]. Although CCND1 gene amplification connectsvery well with increased expression of estrogen protein which suggests some other mechanisms (like p53, p21cip1 pathway) may also contribute to increase cyclin D1 over expression [27].

\section{CYCLIN D1 POLYMORPHISMS AND BREAST CANCER RISK}

It has been reportedin many studies that the CCND1 gene is found over expressed in about $20 \%$ of breast cancerpatients[14, 18]. In exons 4 , there is substitution from $\mathrm{G}$ to A (rs603965) nt870. Some studiesreported that CCND1 forms an alternative protein known as transcript b.Some researchers also evaluated that transcriptb was not a goodfactor of retinoblastoma (RB)activation or inactivation and henceenhanced cell transformation as compared to transcripta[18]. 
Till date, different studies revealed the role of the CCND 1 as riskof breast carcinoma. But the results remain disputable[28].Cui et al.(2012)found AA genotype of the cyclin D1 polymorphism was associated with risk of breast cancer development [29]. However, few studies did not find any association with genotype GA of CCND 1 and risk of breast cancer [30]. Some studies found that AA genotype of cyclin D1 polymorphism were significantly related with breast carcinoma development in Caucasians women, but not in Asian women [31]. These results showed that allelic polymorphisms may increase the breast cancer risk. This effect may differ by ethnicity. Results of some studies are shown in table 1.

Yaylim et al. (2009) reported that frequencies of AG, AA, and GG genotypes were $48.4 \%, 28.1 \%$ and $23.4 \%$ respectively in healthy controls, and $21.1 \%, 39.5 \%$ and $39.5 \%$ resply in breast cancer patients [30]. Statistical significant difference of distribution of percentage frequency of CCND 1 genotype was found among breast cancer patients and control cases. Their findings indicated that patients having $\mathrm{AG}$ genotype have reduced risk of breast cancer [30].

In contrast, Bedewy et al. (2013) found that breast cancer patients with GG genotype produce high amount of transcript $b$, while patients with AA genotype produce more transcript ' $a$ ' [32]. In a study, it was noted that there was a statistical significant variation in the genotype frequency distribution between breast cancer patients and controls. These findings suggest that CCND 1 polymorphism association with breast cancer susceptibility. These findings were consistent with previous findings suggested CCND 1genotype association with breast cancer development $[33,34]$.

Some large scale case control studies of breast cancer observed no correlation between CCND 1 polymorphism and risk of development of breast cancer [28]. Shah et al. found that cyclin D1 polymorphism confer breast cancer in young women of China [33]. These results revealed that patients with AA and AG genotypes were at high risk for breast cancer in contrast those having GG genotype [32, 35].

Some studies associated the cyclin D1 GG genotype with elevated risk of breast cancer but results remain controversial [16]. Some researchers evaluated that, breast cancer breast cancer patients having GG genotype whose relatives suffered from any kind of cancer history showed two fold times greater occurrence of breast cancer than those with AG and AA genotype as compare to controls $(p=0.073)[30]$.

TABLE 1. GENOTYPE DISTRIBUTION OF CYCLIN D1 G870A GENE POLYMORPHISM IN DIFFERENT STUDIES.

\begin{tabular}{|c|c|c|c|c|c|}
\hline \multirow[t]{2}{*}{ S.No } & \multirow{2}{*}{$\begin{array}{l}\text { Reference } \\
\text { (Country/year) }\end{array}$} & \multirow{2}{*}{$\begin{array}{l}\text { No. of patients } \\
\text { (sample size) }\end{array}$} & \multicolumn{2}{|c|}{ Genotype distribution (\%) } & \multirow[b]{2}{*}{ AA } \\
\hline & & & GG & GA & \\
\hline 1 & Krippl 2003 & 497 & 23 & 49 & 28 \\
\hline 2 & Grieu 2003 & 339 & 27 & 48 & 25 \\
\hline 3 & Forsti 2004 & 223 & 26 & 52 & 22 \\
\hline 4 & Ceschi 2005 & 255 & 23 & 38 & 40 \\
\hline 5 & Shu 2005 & 1130 & 19 & 50 & 31 \\
\hline 6 & Naidu 2008 & 230 & 25 & 45 & 30 \\
\hline 7 & Onay 2008 & 1228 & 30 & 48 & 21 \\
\hline 8 & Justenhoven2009 & 1143 & 24 & 48 & 28 \\
\hline 9 & Yaylim 2009 & 38 & 39 & 22 & 39 \\
\hline 10 & Canbay2010 & 78 & 13 & 61 & 26 \\
\hline 11 & Jeon2010 & 769 & 24 & 47 & 29 \\
\hline 12 & Bedewy 2013 & 30 & 14 & 60 & 26 \\
\hline 13 & Liu 2014 & 1232 & 15 & 60 & 26 \\
\hline 14 & Soleimani 2017 & 174 & 21 & 50 & 28 \\
\hline 15 & Thakur 2018 & 151 & 33 & 77 & 41 \\
\hline 16 & Akhter 2019 & 30 & 14 & 26 & 60 \\
\hline
\end{tabular}

In lymph node metastasis, breast cancer cases having the cyclin D1 GG genotype had around two fold increased breast cancer risk as compared with those with the $A G$ and AA geno types.In a study, it was observed that the' $A$ ' allele of the CCND 1 polymorphism was weakly related with risk of breast cancer among women ages <45 years[12]. It was also found that CCND1 polymorphism may changes the association of endogenous sex hormone exposure with postmenopausal risk of breast cancer development. Among women with the AA or AG genotype, BMI and WHR, as well as blood levels of testosterone and esterone were positively associated with postmenopausal breast cancer risk. These variables were inversely proportional to risk of breast cancer in women having GG genotype[11].

In addition it was also found that the inverse association between SHBG level and postmenopausal breast cancer risk was restricted only to women carrying the A allele of the CCND 1 polymorphism. Furthermore, CCND 1 polymorphism was also found correlated with a favorable outcome for those women who showed later stages of cancer or negative expression of ER or PR (estrogen and progesterone receptor). 


\section{ETHNICITY}

Some recent studies reported statistically significant association between CCND1 polymorphism and increased breast cancer risk in carriers of variant $870 \mathrm{~A}$ allele in Caucasians women and not in Asian women. They proposed that environmental exposures and here dity might also contribute to differences in ethnicity. Although some studies could not found significant result for homozygous A or G alleles [30].Yaylim et al. (2009) found that women with $\mathrm{AG}$ genotype have a reduced risk of breast cancer development [36]. Few studies found no relation of GA genotype with development of breast cancer risk.

Further large scale case control breast cancer studies have observed no statistically significant correlation between CCND1 polymorphism and risk of breast cancer on women populations including Singapore, Chinese, Australian, Austrian, German, Finnish and Malaysian populations [28]. On the other side, 'GA' genotype of CCND1 found weak association with risk of breast carcinoma. The A Agenotype and Aallele showed similar prevalence among breast cancer patients and controls $[33,34]$.

\section{CLINICAL RELEVANCE}

There are many studies that have suggested, cyclin D1 amplification linkage with poor response of tamoxifen $[36,37]$. Generally estrogen receptor positive breast tumors show cyclin D1 over expression [38].Transcriptional activity of the estrogen receptor is suggested to be regulated by cyclin D1 in absence of estrogen $[39,40]$. That is why its expression is also affected by some antiestrogen drugs [41].

There are certain evidences that suggest cyclin dependent kinase, their substrates and regulators as targets of genetic mutation in different kinds of human cancer.These encourage chemical CDK inhibitorsas anticancer drugs [36]. Different strategies for therapeutic interferencecan modify CDK activity: targeting the important regulators of CDK activity or interfering the catalytic activity of CDKs. Approaches for the indirect methods include overexpression of CKI, synthesis of peptides which resembles the effects of CKI, decrease level of cyclin, modifications in proteasomal machinery and change in phosphorylated state of CDK and enzymes which regulate it [41].

\section{CONCLUSION}

A large number of studies have pointed out the role of CCND1gene polymorphism in many aspects of breast cancer, but the functional impact of this polymorphism is not fully understood. Further, correlation among the CCND1 polymorphism and expression of transcript variants $\mathrm{a}$ and $\mathrm{b}$ are required to investigate and how polymorphism is involved in development of breast cancer. In future functional studies may help in explaining the conflicting findings and effect of CCND1 genotypes on tumour behaviour in different cell types.

\section{ACKNOWLEDGMENT}

The Authors are grateful to department of biotechnology, Chandigarh College of technology,
Landran, 140307, Mohali, Punjab, India for providing help and support

COMPETING INTERESTS: Nil

\section{REFERENCES}

[1]. A. Jemal, R. Siegel, E. Ward, Y. Hao, and J. Xu. 2009, “Cancer statistics," CA Cancer J Clin, vol. 5, pp. 225249, 2009.

[2]. Lim, S and Kaldis, P. "Cdks cyclins and CKIs: roles beyond cell cycle regulation," Development, vol.140.15, pp. 3079-3093,2013.

[3]. Whittaker,S.R. Mallinger,A. Workman,P. and Clarke, P.A. "Inhibitors of cyclin-dependent kinases as cancer therapeutics," Pharmacology \& therapeutics, vol. 173, pp. 83-105,2017.

[4]. Wood, D. J. and Endicott, J. A. "Structural insights into the functional diversity of the CDK-cyclin family," Open biology,vol. 8.9, pp.180112,2018.

[5]. Duronio, R. J. andXiong, Y. "Signaling pathways that control cell proliferation," Cold SpringHarbor perspectives in biology, vol.5.3, pp. 008904,2013.

[6]. Wasson, M. K. Chauhan, P. S. Singh,D.Katara, L. C. Sharma, J.and Zomawia, E. "Association of DNA repair and cell cycle gene variations with breast cancer risk in Northeast Indian population: a multiple interaction analysis," Tumour Biol,vol. 35, pp. 5885-5894,2014.

[7]. Thakur, N. Kumari, S. and Mehrotra, R. "Association between Cyclin D1 G870A (rs19344) polymorphism and cancer risk in Indian population: meta-analysis and trial sequental analysis," Bioscience reports,vo. 38.6,2018.

[8]. Zhang, Y. Zeng, X. Lu,H.Ji, and Zhao, E. "Association between cyclin D1 (CCND1) G870A polymorphism and gastric cancer risk: a meta-analysis,"Oncotarget, vol. 7, pp. 66109-66118,2016.

[9]. Xu, X. M. Ni, X. B. Yang, G. L. Luo, Z. C. and Shen, M "CCND1 G870A polymorphism and colorectal cancer risk: an updated meta-analysis," Mol. Clin. Oncol, vol. 4, pp. 1078-1084,2016.

[10]. Malumbres, M. "Cyclin-dependent kinases," Genome biology, vol. 15.6, pp. 10,2014.

[11]. Canbay, E. Eraltan, I. Y. Cercel, A.and Isbir, T. "CCND1 and CDKN1B polymorphisms and risk of breast cancer," Anticancer research,vol. 30.7, pp. 3093-3098,2010.

[12]. Gyorffy, B. Lanczky, A. and Eklund, A. C. "An online survival analysis tool to rapidly assess the effect of 22,277 genes on breast cancer prognosis using microarray data of 1,809 patients," Breast cancer research and treatment,vol. 123.3, pp.725-731,2010.

[13]. Lu, Y. Gao, K. Zhang, M. Zhou, A. and Ge, S. "Genetic association between CDKN1B rs2066827 polymorphism and susceptibility to cancer," Medicine,vol. 94.46,2015.

[14]. Lin, Z. Zhang, X Zhao, F. and Ru, S. "Bisphenol S promotes the cell cycle progression and cell proliferation through ER $\alpha$-cyclin D-CDK4/6-pRb pathway in MCF-7 breast cancer cells," Toxicology and Applied Pharmacology, vol.366, pp.75-82,2019.

[15].Maghvan,P.V. Rezaei-avirani, M. H.Zali, Khodadoostan, andH.Asadzadeh-Aghdaei, M. "Network analysis of common genes related to esophageal, gastric, and colon cancers," Gastroenterology and Hepatology from bed to bench, vol.10.4, pp.295,2017.

[16]. Chen, B. Cao, L Yang, PZhou. Y. and Wu, X. T. "Cyclin D1 (CCND1) G870A gene polymorphism is an ethnicitydependent risk factor for digestive tract cancers: a meta- 
analysis comprising 20,271 subjects," Cancer epidemiology, vol. 36.2, pp.106-115,2012.

[17]. Qie, S. andA. Diehl, J. "Cyclin D1, cancer progression, and opportunities in cancer treatment," Journal of Molecular Medicine,vol. 94.12, pp. 1313-1326,2016.

[18]. Slotta-Huspenina, J. Koch, I. L. de Leval, Keller, G. Klier, M. and Bink, K. "The impact of cyclin D1 mRNA isoforms, morphology and p53 in mantle cell lymphoma: p53 alterations and blastoid morphology are strong predictors of a high proliferation index,"'haematological, vol.97.9, pp. $1422-1430,2012$.

[19]. Shlien, A. Raine, K. Fuligni, F.. Arnold, R Nik-Zainal, S. and Dronov, S. "Direct transcriptional consequences of somatic mutation in breast cancer," Cell reports, vol. 16.7, pp. 2032-2046,2016.

[20]. Lundgren, K. Brown, M. Pineda, S. Cuzick, J. and Salter, J. "Effects of cyclin D1 gene amplification and protein expression on time to recurrence in postmenopausal breast cancer patients treated with anastrozole or tamoxifen: a TransATAC study," Breast Cancer Research, vol.14, pp.R57,2012.

[21]. Ravikumar, G. and Ananthamurthy, A. "Cyclin D1 expression in ductal carcinoma of the breast and its correlation with other prognostic parameters," Journal of cancer research and therapeutics, vol. 10.3, pp. 671,2014.

[22]. Sarkar, S. Kanoi, A. Bain, J. and Das, K. N. "Correlation between cyclin D1 expression and standard clinicopathological variables in invasive breast cancer in Eastern India," South Asian journal of cancer, vol. 4.4, pp. 155,2015 .

[23]. Peurala, E. Koivunen, P. M.Haapasaari, K. and JukkolaVuorinen, A. "The prognostic significance and value of cyclin D1, CDK4 and p16 in human breast cancer," Breast Cancer Researc, vol. 15.1, pp. R5,2013.

[24]. Li, Z. Cui, J. Yu, Q. Wu, X. andPan, A. "Evaluation of CCND1 amplification and CyclinD1 expression: diffuse and strong staining of CyclinD1 could have same predictive roles as CCND1 amplification in ER positive breast cancers," American journal of translational research, vol. 8.1, pp.142,2016.

[25]. John, R. Malathi, N. Ravindran, C. and Anandan, S. "Mini review: Multifaceted role played by cyclin D1 in tumor behaviour," Indian Journal of Dental Research, vol.28.2, pp.187,2017.

[26]. Mrhalova, M. Kodet, R. and Strnad, P. "Invasive ductal carcinoma of the breast: Study of the number of copies of the CCND1 gene and chromosome 11 using fluorescence in situ hybridization (FISH) in comparison with expression of cyclin D1 protein and estrogen receptors (ER alpha) with immunohistochemical detection,"CasLekCes, vol.141, pp.708-714,2002.

[27]. Jirstrom, K. Stendahl, M. and Rydén, L. "Adverse effect of adjuvant tamoxifen in premenopausal breast cancer with cyclin D1 gene amplification," Cancer Res, vol.65, pp. $8009-8016,2005$

[28]. Sergentanis, T. N. and Economopoulos, K. P. "Cyclin D1 G870A polymorphism and breast cancer risk: a metaanalysis comprising 9,911 cases and 11,171 controls," Molecular biology reports, vol.38.8, pp. 4955-4963,2011.

[29]. Cui, J. Shen, L. and Wang, Y. "Specific CCND1 G870A alleles associated with breast cancer susceptibility: a metaanalysis of 5,528 cases and 5,353 controls," Asian Pacific
Journal of Cancer Prevention, vol. 13.10, pp. 50235025,2012 .

[30]. Yaylim-Eraltan, I. Ergen, A. Görmüş, U. and Şahin, O. "Breast cancer and cyclin D1 gene polymorphism in Turkish women," in vivo, vol. 23.5, pp. 767-772,2009.

[31]. Akhter, N. Alzahran, F. A. Dar, S. A. Wahid, M. R. Sattar, S. and Almalki, S. "AA genotype of cyclin D1 G870A polymorphism increases breast cancer risk: Findings of a case-control study and meta-analysis," Journal of cellular biochemistry, vol.120.10, pp.16452-16466,2019.

[32]. Bedewy, A. M. Mostafa, M. H. Saad, A. A. El-Maghraby, S. M. and Kandil, L. S. "Association of cyclin D1 A870G polymorphism with two malignancies: acute lymphoblastic leukemia and breast cancer," J BUON, vol. 18.1, pp. 227 238,2013 .

[33]. Shah, A. U. Mahjabeen, I. and Kayani, M. A. "Genetic polymorphisms in cell cycle regulatory genes CCND1 and CDK4 are associated with susceptibility to breast cancer," J buon, vol. 20, pp. 985-983,2015.

[34]. Rai, V. Yadav, U. and Kumar, P. "Impact of catechol-Omethyltransferase Val 158Met (rs4680) polymorphism on breast cancer susceptibility in Asian population," Asian Pacific Journal of Cancer Prevention: APJCP, vol. 18.5, pp.1243,2017.

[35]. Liu, L. C. Su, C. H. Wang, H. C. Chang, W. S. and C. W,"Contribution of personalized Cyclin D1 genotype to triple negative breast cancer risk," Biomedicine, vol.4.1,2014.

[36]. Soleimani, Z. Kheirkhah, D. Sharif, M. R. Sharif, A. and Aftabi, Y. "Association of CCND1 Gene c. 870G> A polymorphism with breast cancer risk: A case-controlstudy and a meta-analysis," Pathology \& Oncology Research, vol. 23.3, pp. 621-631,2017.

[37]. Bostner, J. AhnstromWaltersson, M. Fornander, T. and Skoog, L. "Amplification of CCND1 and PAK1 as predictors of recurrence and tamoxifen resistance in postmenopausal breast cancer," Oncogene, vol. 26.49, pp. 6997-7005,2007.

[38]. Ortiz, A. B. Garcia, D. Vicente, Y. Palka, M. and Martin, P. "Prognostic significance of cyclin D1 protein expression and gene amplification in invasive breast carcinoma,"PloS one, vol.12.11, pp. e0188068,2017.

[39]. Chauhan, P. Babu, M. A. Kaur, S. P. and Menon, V. "Hereditary Breast Cancer: A Systematic Review," CGCIJCTR, vol. 2.1, pp. 48-52,2019.

[40]. Ravikumar, G. and Ananthamurthy, A. "Cyclin D1 expression in ductal carcinoma of the breast and its correlation with other prognostic parameters," J Cancer Res Ther, vol. 10.3, pp. 671-675,2014.

[41]. Burandt, E. Grünert, M. Lebeau, A. Choschzick, M.and Geist, S. "Cyclin D1 gene amplification is highly homogeneous in breast cancer," Breast Cancer, vol.23.1, pp.111-119,2016. 